\title{
Requerimiento hídrico y edad de trasplante del calabacín (Cucúrbita pepo L.) bajo riego por goteo en el Valle del Rio Guarapiche
}

\section{Water requirement and transplanting age of zucchini (Cucúrbita pepo L.) under drip irrigation in the Guarapiche River Valley}

\author{
José Alexander Gil-Marín ${ }^{1 *}$ (D), María Ximena Cordova-Rodriguez² (D), Nelson José Montaño-Mata ${ }^{1}$
}

\section{RESUMEN}

El presente trabajo se realizó en el sector San Vicente, Municipio Maturín Estado Monagas, Venezuela entre los meses de Junio-Septiembre del 2015, con el objetivo de determinar el efecto de cuatro láminas de riego y dos edades de trasplante sobre las variables físicas de número de frutos, longitud, diámetro y peso del fruto en el cultivar Shiraz de calabacín (Cucurbita pepo L.). Se utilizó un diseño experimental de bloques al azar con tres repeticiones, con arreglo de parcelas divididas, donde la parcela principal fueron las diferentes láminas de riego y las subparcelas las dos edades de trasplante. Se aplicaron 8 tratamientos que resultaron de la combinación de las edades de trasplante, edad 1 (14 días) y la edad 2 (21 días) y cuatro criterios de riego consistentes en reponer el equivalente al $60 \%, 80 \%$, $100 \%$ y $120 \%$ de la evapotranspiración del cultivo (ETc). Un tanque evaporímetro tipo "A" se utilizó para estimar la evapotranspiración del calabacín. De los resultados obtenidos se concluye que la lámina de riego y la edad de trasplante que más efecto tuvo en la calidad de los frutos de calabacín fue la lámina de $120 \%$ de ETc y la edad de trasplante 2 (21 días). Se confirma el efecto negativo de las condiciones de déficit de riego sobre los atributos de calidad física. En este trabajo las variables más afectadas fueron peso y número de frutos.

Palabras claves: eficiencia del uso del agua, evapotranspiración, riego por goteo, calidad del fruto.

\begin{abstract}
The present work was carried out in San Vicente sector, Maturín Municipality, Monagas State, Venezuela between the months of June-September 2015, with the objective of determining the effect of four irrigation deeps and two transplant ages on the physical variables of number of fruits, length, diameter and weight of fruit in the cultivar Shiraz of squash (Cucurbita pepo L.). A randomized block design with three replications was used, with a divided plot arrangement, where the main plot was the different irrigation deep and the subplots the two transplant ages. 8 treatments were applied that resulted from the combination of the transplant ages, age 1 ( 30 days) and age 2 (40 days) and four irrigation criteria consisting of replacing the equivalent of $60 \%, 80 \%, 100 \%$ and $120 \%$ of crop evapotranspiration (ETc). Class A pan was used to estimate the eggplant evapotranspiration. The results showed that the irrigation deep and the transplant age that had the most effect on the quality of the squash plant fruits was the deep of $120 \%$ ETc and the transplant age 2 ( 21 days). The negative effect of the irrigation deficit conditions on the physical quality attributes is confirmed, in this work the most affected variables were weight and number of fruits.
\end{abstract}

Keywords: water use efficiency, evapotranspiration, drip irrigation, fruit quality.

\footnotetext{
Universidad de Oriente, Departamento de Ingeniería Agrícola, Maturín, Venezuela

${ }^{2}$ Universidad Nacional de Loja, Facultad de Salud Humana, Loja, Ecuador

"Autor de correspondencia. E-mail:jalexgi12005@hotmail.com
} 


\section{INTRODUCCION}

El cambio climático global y la creciente competencia por el agua dulce pueden tener graves impactos en los recursos hídricos y el sector agrícola en un futuro próximo. El acceso al agua de riego es clave para reducir los impactos de la variabilidad y el cambio climático en la seguridad alimentaria. El riego es una práctica cada vez más importante para la agricultura sostenible en las regiones áridas y semiáridas (Fernández et al., 2020). Para resolver el problema de la escasez de agua en la agricultura es necesario desarrollar tecnologías de gestión de ahorro de agua. La aplicación de métodos de riego modernos y tecnologías acompañantes es un concepto importante que debe hacerse en las zonas áridas y semiáridas para ahorrar una parte del agua de riego (Abdelraouf et al., 2012; El-Habbasha et al., 2014). Venezuela ha estado sufriendo escasez de agua en los últimos años por efectos del cambio climático, pero sus fuentes de agua todavía no son limitadas en comparación con la creciente demanda de agua. Por lo tanto, ajustar la gestión del agua tanto en tierras nuevas como antiguas es un componente importante del desarrollo agrícola. La agricultura de regadío es el principal contribuyente de la producción agrícola, y se enfrenta al desafío de mejorar la eficiencia del uso del agua de riego y, al mismo tiempo, garantizar la seguridad alimentaria (Li et al., 2016). El consumo mundial de agua para riego ha crecido constantemente durante los últimos 50 años y hoy representa el $70 \%$ de todo el consumo de agua (Tian et al., 2017). El gran desafío del sector agrícola es producir más alimentos con menos agua, lo que se puede lograr aumentando la productividad del agua de los cultivos (CWP) (Zwart y Bastiaanessen, 2004). La deficiencia de agua dulce aumentó significativamente en todo el mundo. Según las previsiones de la FAO y el IFPRI, la demanda mundial de recursos hídricos según el escenario de desarrollo habitual para el 2030 se duplicará. La mejora de los sistemas de riego agrícola se considera una parte importante del desarrollo de la agricultura. La razón principal de tal demanda de agua es la alta producción de la masa aérea de hojas con un alto coeficiente de transpiración (Okasha et al.,
2020). Generalmente, el riego de hortalizas cucurbitáceas afecta claramente tanto al rendimiento como a la calidad de la fruta. Los estudios de Peil et al. (2012) y Yavuz et al. (2015), señalan que, con el aumento de la cantidad de riego, las características de rendimiento aumentaron significativamente. Por otro lado, aunque el riego suele incrementar el rendimiento, también suele provocar una disminución de la composición química del fruto. El uso mundial de sistemas de riego por goteo superficial y subterráneo ha aumentado considerablemente en las últimas décadas. El riego por goteo puede lograr una alta eficiencia en el uso del agua, pero solo cuando el sistema está diseñado correctamente, con el espaciamiento de emisores, la tasa de flujo y la profundidad de instalación adecuados (Rafie y El-Boraie, 2017). Hoy en día, el sistema de riego por goteo, que entrega agua directamente a la zona de raíces de las plantas, es una de las soluciones más eficientes y económicas para suministrar agua a estos seres vivos. Además, mediante el riego por goteo, gran parte del suelo fuera de la zona radical permanece seco, por lo que son menos susceptibles a las infecciones bacterianas o fúngicas. Este sistema se adapta perfectamente a hortalizas como el calabacín. El calabacín es considerado uno de las hortalizas más importantes del mundo por ser un cultivo comercial para campos e invernaderos. Se produce en la mayoría de los países como una de las principales hortalizas y también es ampliamente cultivada y consumida en Venezuela. El suministro de agua es uno de los factores más importantes que puede afectar en gran medida el rendimiento y la calidad del calabacín. Esta hortaliza se cultiva predominantemente en campos pequeños que tienen menos de una hectárea en las temporadas de primavera, verano y otoño. Por lo tanto, el método de riego por goteo es beneficioso para su productividad agrícola (Ferreyra y Jeznach, 2007; Rolbiecki, 2017). Ibrahim y Selim (2007;2010) concluyeron que el riego con intervalos de 12 días en el Cucurbita pepo cv Eskandrani aumentó la eficiencia del uso del agua y produjo un mayor rendimiento de fruta, satisfactorio y comercializable. Mientras El-Gindy et al. (2009) señalan que el riego por goteo subterráneo 
tiene una mejor distribución del agua de riego en el suelo, lo que considera más adecuado para las raíces y el rendimiento del calabacín. Aunque hay muchas investigaciones sobre las influencias de las limitaciones regulada del agua en los parámetros de rendimiento y calidad, hay relativamente pocos trabajos relacionados con el riego de calabacín en las condiciones locales del oriente del país y Maturín. Por lo tanto, el objetivo de esta investigación fue determinar las influencias de diferentes niveles de riego y edades de trasplante sobre la calidad de los frutos del cultivo de calabacín.

\section{MATERIALES Y METODOS}

El trabajo se realizó en el sector de San Vicente, municipio Maturín, estado Monagas, Venezuela, entre los meses de Marzo y Septiembre del 2015. Su ubicación geográfica está comprendida entre los $9^{\circ} 44^{\prime} 37^{\prime \prime}$ de latitud Norte y $\operatorname{los} 63^{\circ} 15^{\prime} 59^{\prime \prime}$ de longitud Oeste, a una altitud de 51 m.s.n.m (Figura 1). El clima de la zona según Holdridge y citado por Montaño et al. (2018), es del tipo Bosque Seco Tropical, caracterizado por presentar una estación lluviosa de Mayo a Diciembre y una estación seca de Enero a Abril, con una precipitación media anual de 1219,6 mm, una temperatura media anual de $25,9^{\circ} \mathrm{C}$, con una evapotranspiración potencial de $1372 \mathrm{~mm}$ y una evaporación de $1573 \mathrm{~mm}$ al año.

El suelo predominante es de textura franco arenosa y se clasifican taxonómicamente como ultisol (paleustults), que se caracteriza por ser muy lixiviado, con $\mathrm{pH}$ de 4,7; baja capacidad de intercambio catiónico y bajo porcentaje de materia orgánica (Gil et al., 2014) (Tabla 1).

Se utilizó el cultivar de calabacín Shiraz a un espaciamiento de $0,3 \mathrm{~m}$. El experimento se presentó en un diseño de parcela dividida con tres repeticiones (Figura 2) y ocho tratamientos producto de cuatro niveles del factor riego como parcela principal $(60,80,100 \mathrm{y}$ $120 \%$ ETc) y dos niveles de la edad de trasplante como sub-parcela (14 y 21 días). La programación del riego se realizó en días alternos sobre la base de la evapotranspiración del cultivo de referencia (ETo) y los coeficientes del cultivo $(\mathrm{Kc})$. La evapotranspiración del cultivo de referencia (ETo) se estimó utilizando el Método de la Tina de Evaporación Tipo A de la FAO. La lectura diaria de evaporación registrada en la tina

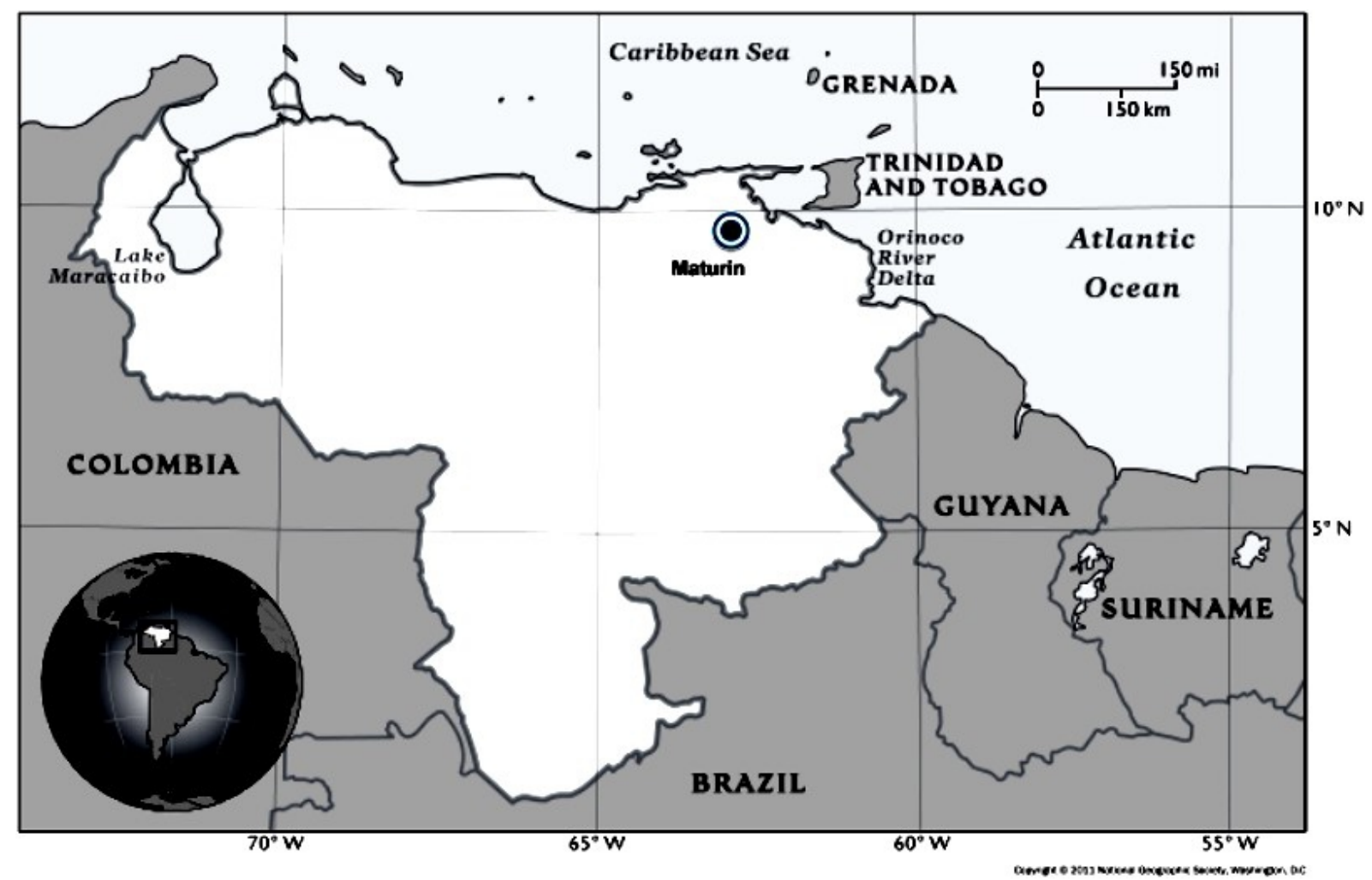

Figura 1. Ubicación geográfica de la Ciudad de Maturín. Estado Monagas. Venezuela. Fuente: Gil et al. (2012). 
Tabla 1. Algunas propiedades físicas y químicas del suelo donde se desarrolló el trabajo.

\begin{tabular}{ccc}
\hline Características & Valor & Método \\
\hline Textura & Fa & Bouyoucos \\
pH & 4,7 & Potenciómetro \\
Materia Orgánica (\%) & 3,37 & Walkley y Blank \\
CE (micro S/m) & 192,4 & Conductimetro \\
\hline
\end{tabular}

Análisis realizado en el laboratorio de suelos de la Universidad de Oriente, Núcleo Monagas, Campus los Guaritos. (Gil et al. 2014).

clase A se convirtió a ET de referencia (ETo) al multiplicar por el coeficiente de la tina $(\mathrm{Kp})$ de $(0,80)$; valor obtenido después de considerar la humedad relativa y la velocidad del viento predominante de la zona. Por último, la ET del cultivo (ETc) se obtuvo multiplicando ETo por el coeficiente del cultivo $(\mathrm{Kc})$ : $\mathrm{ETc}=\mathrm{ETo} \times \mathrm{Kc}$, como lo recomienda Allen et al., (1998). Los valores de Kc locales estimados para riego por goteo y calabacín en este estudio fueron 0,$3 ; 0,6 ; 0,90$ y 0,75 para las diferentes etapas del cultivo. Cada etapa duro aproximadamente 25 días. Las parcelas estuvieron constituidas por cuatro laterales de $10 \mathrm{~m}$ de longitud, separadas a $1 \mathrm{~m}$ entre ellos, con goteros de riego cada $0,33 \mathrm{~m}$, considerándose para efectos de evaluación solo las dos hileras centrales. Se instaló un sistema de riego por goteo con goteros integrados tipo Flat dripper line marca Aquadrop, con un espesor de pared de 0,3 mm y un caudal de $1,0 \mathrm{~L} / \mathrm{h}$. El área total del experimento fue de $448 \mathrm{~m}^{2}$, teniendo las parcelas principales un área de $30 \mathrm{~m}^{2}$ y las sub-parcelas de $5 \mathrm{~m}^{2}$. La preparación del terreno, se realizó con tres pases de rastra, con el objetivo de eliminar las malezas presentes y dar al suelo una soltura que permitiera el desarrollo del sistema radical del cultivo y se surco el terreno, con separación de 1,00 m. Las plántulas fueron obtenidas en bandejas de germinación de 162 alvéolos, y el sustrato utilizado fue una mezcla de turba y sustrato de corteza de pino (PROFORCA), en una proporción de 3:1, realizándose la siembra de la primera edad (14 días después de la germinación, ddg y 18 días después de la siembra, dds), el día 15 de abril de 2015 y la segunda edad (21 días después de la germinación, ddg y 25 días después de la siembra, dds) el 22 de abril de 2015. La cantidad de semillas utilizada fue de 1 semillas por alvéolo para un total máximo de 162 semillas por bandeja y 2916 por todo el ensayo. La germinación se inició a los 4 días luego de la siembra. La frecuencia de riego de las plántulas fue de dos veces al día, aplicándose una en la mañana y la otra en la tarde. El control de malezas, cuando la incidencia de estas lo requirió, se efectuó de forma manual o con herbicida. Asimismo, se realizó un apropiado control fitosanitario del ensayo. La fertilización se realizó a través del sistema de riego usando diariamente fertilizantes solubles de fórmula 27,5-49-0 durante los primeros 30 días y la formula 28-14-30 durante el resto de la etapa del cultivo. La cantidad de fertilizante aplicado estuvo en el rango de 200-500 g.dia ${ }^{-1}$. Se hicieron los ajustes necesarios en el sistema con el fin de mantener iguales las dosis aplicadas por planta, independientemente de los diferentes volúmenes de empleados en cada tratamiento de riego.

Los calabacines de cada parcela unitaria fueron cosechados y a los frutos se le determino las variables longitud del fruto $(\mathrm{cm})$, diámetro del fruto $(\mathrm{cm})$, número de frutos y peso de frutos frescos. Los análisis estadísticos fueron realizados utilizando el Proc GLM (modelos lineales generales) procedimiento de SAS (SAS Institute, Inc., Cary, N. C.) en un nivel de significancia de $p \leq 0,05$. El análisis de varianza (ANOVA) se realizó para determinar las diferencias entre tratamientos para cada parámetro como aplicable al diseño completo de bloques al azar dispuestos en procedimiento de parcelas divididas. Las medias de tratamiento se compararon con el Procedimiento de Duncan a 0,05 nivel de probabilidad.

\section{RESULTADOS Y DISCUSIÓN}

Efecto de los tratamientos de riego sobre los componentes del rendimiento del calabacín

Las propiedades físicas de la fruta, como número de frutos, longitud, el diámetro, el peso $\mathrm{y}$, en última instancia, el rendimiento, se consideran los parámetros más importantes a considerar en la obtención de los 
Tabla 2. Análisis de varianza de las variables número de frutos, longitud, diámetro y peso del fruto en la cosecha total del cultivar de calabacín Shiraz, bajo el efecto de cuatro estrategias de riego y dos edades de trasplante, estudiados en el sistema de riego Perú-San Vicente del rio Guarapiche Maturín-Monagas.

\begin{tabular}{cccccc}
\hline $\begin{array}{c}\text { Fuente de } \\
\text { Variación }\end{array}$ & $\begin{array}{c}\text { Grados de } \\
\text { Libertad }\end{array}$ & $\begin{array}{c}\text { Numero de } \\
\text { Frutos }\end{array}$ & $\begin{array}{c}\text { Longitud } \\
\text { del Fruto (cm) }\end{array}$ & $\begin{array}{c}\text { Diámetro } \\
\text { del Fruto (cm) }\end{array}$ & $\begin{array}{c}\text { Peso del } \\
\text { Fruto (g) }\end{array}$ \\
\hline Bloque & 2 & 3.78 & 2.71 & 3.27 & 3.74 \\
Riego (A) & 3 & $23.19 * *$ & $158.51 * *$ & $147.46 * *$ & 0.36 \\
Error (A) & 6 & 0.53 & 0.43 & $6.36^{* *}$ & 1.48 \\
Edad (B) & 1 & $6.61 *$ & $1.18 \mathrm{~ns}$ & $2.15 \mathrm{~ns}$ & 0.006 \\
Riego* Edad & 3 & $3.04 \mathrm{~ns}$ & 0.11 & $1.66 \mathrm{~ns}$ & 0.11 \\
Error (B) & 8 & 13.92 & 1.65 & 8.05 \\
CV (\%) & & 17.94 & & 1.51 \\
\hline
\end{tabular}

* Significativo al 5\%;** altamente significativo al 1\%; ns no significativo.

mejores beneficios económicos para los agricultores.

El efecto negativo de las condiciones de déficit de riego sobre estos atributos de calidad física está bien establecido y estudiado por Colak et al. (2015) en berenjena, y Pervez et al. (2009) en tomates. En la Tabla 2 se muestran los resultados del análisis de varianza para todas las variables físicas de estudio, donde se observan los efectos altamente significativos y significativos de los factores riego y edad, respectivamente, y no significativos para su interacción.

\section{Número de frutos por planta}

En la Tabla 3 se observa que el número de frutos por planta fue afectado por los niveles de agua de riego, con niveles de significancia altamente significativos menores al $\mathrm{p} \leq 0,01$. El tratamiento de $120 \%$ de ETc registró el mayor número de frutos por planta con un promedio de 2,75, seguido de la lámina de riego del $100 \%$, con promedio de 2,25. El menor número de frutos por parcela se observó en las estrategias de riego 80 y $60 \%$ ETo, con promedios de 1,75 y 1,57 frutos, respectivamente, sin diferencias significativas entre ambos. El cambio de la dosis de riego de $120 \%$ a $60 \%$ ETc, disminuyó en un 42,9\% el número de frutos, lo que evidencia el efecto negativo del estrés hídrico en este cultivo. Esto posiblemente se debe al hecho de que el agua aplicada a $120 \%$ ETc cumple de manera adecuada con los requerimientos hídricos del cultivo. El número de flores $\mathrm{y}$, en última instancia, el número de frutos por planta son parámetros económicos importantes, ya que afectan la producción final y, por tanto, la economía del agricultor, por lo que cualquier estrés que reduzca el número de frutos por planta debe mane- jarse adecuadamente. Los parámetros reproductivos se ven afectados por el estrés hídrico, reduciendo la transpiración y fotosíntesis, lo que resulta en una reducción del número de flores por planta, cuajado y frutos producidos. Los resultados de esta investigación concuerdan con los hallazgos de Alzoheiry y AlMoshileh (2018) y Kuslu et al. (2014), que indican que el rendimiento de las plantas y sus componentes disminuyeron con el aumento del déficit hídrico. Bafeel y Moftah, (2008), reportan que el efecto negativo del estrés por sequía sobre el rendimiento y sus componentes puede estar relacionado con la disminución del crecimiento vegetativo.

El promedio general del número de frutos por plantas en este experimento fue de 2,08; valor muy semejante al reportado por Wetzel y Stone (2019) en la variedad Sunshine con 2,28 frutos por planta, pero por debajo de los valores reportados por Moreno-Rosendez et al. (2019), con 10,1 en condiciones de invernadero y con el hibrido Mona Lisa F1.

\section{Longitud del fruto}

El cuadro 2 resume los resultados del análisis de varianza. Los resultados de la prueba son significativos a una probabilidad de 0,01 para el efecto de niveles de agua de riego en la longitud de los frutos, indicando que la mayor longitud de frutos se obtuvo con el tratamiento $120 \%$ ETc, seguido de $100 \%$ y $80 \%$ de ETc, mientras que el $60 \%$ de ETc registró el menor valor. El cambio de régimen de riego de 120 a $60 \%$ ETc, trajo en consecuencia una disminución del 12,03\% de la longitud del fruto de calabacín. Esto significa que el contenido de humedad del suelo con una lámina apli- 
Tabla 3. Efecto del régimen de riego sobre las variables de calidad del fruto

\begin{tabular}{ccccc}
\hline $\begin{array}{c}\text { Niveles hídricos } \\
\text { de reiego (\%ETc) }\end{array}$ & $\mathbf{N}^{\circ}$ de frutos & $\begin{array}{c}\text { Longitud del fruto } \\
\text { (cm) }\end{array}$ & $\begin{array}{c}\text { Diámetro del } \\
\text { fruto (cm) }\end{array}$ & Pero del fruto (g) \\
\hline 120 & $2,75 \mathrm{a}$ & $21,53 \mathrm{a}$ & $5,42 \mathrm{a}$ & $595.32 \mathrm{a}$ \\
100 & $2,25 \mathrm{~b}$ & $21,06 \mathrm{~b}$ & $5,24 \mathrm{~b}$ & $504,24 \mathrm{~b}$ \\
80 & $1,75 \mathrm{c}$ & $20,22 \mathrm{c}$ & $5,07 \mathrm{c}$ & $437,16 \mathrm{c}$ \\
60 & $1,57 \mathrm{c}$ & $18,94 \mathrm{~d}$ & $4,88 \mathrm{~d}$ & $392,43 \mathrm{~d}$ \\
DMS & 0,49 & 0,45 & 0,104 & 0,04 \\
CV (\%) & 17,74 & 1,65 & 1,51 & 8,05 \\
\hline
\end{tabular}

Medias seguidas por diferentes letras en una misma columna son estadísticamente diferentes a $\mathrm{p} \leq 0,05$

cada de $120 \%$ de ETc es adecuada para el crecimiento de estas frutas. Estos resultados están en conformidad con los obtenidos por Xu y Leskovar (2014) que afirmaron que el rendimiento y los parámetros de calidad disminuyen cuando el régimen de riego está por debajo del $100 \%$ de la ETc. Kuslu et al. (2014), trabajando con el hibrido de calabacín Eskenderany, con riego por goteo y con diferentes regímenes de riego, en la región semiárida de Egipto reporta un valor promedio de $19,56 \mathrm{~cm}$ de longitud. Por otro lado, El-Mageed y Semida (2015) estudiando el efecto del régimen de riego en el hibrido Hi Tech reporta promedios 14,68 cm. Mientras, Esho y Saeed. (2017), en condiciones áridas de Iraq, trabajando con tres cultivares de calabacín (Alexandria F1, Khatoon F1 y Ardendo), y diferentes combinaciones de ácidos húmicos, reporta valores promedios de 15,32 cm; todos por debajo del promedio observado en este trabajo de $20,44 \mathrm{~cm}$.

\section{Diámetro de las frutas}

El efecto de los niveles de agua de riego en el diámetro de los frutos, monitoreado durante la temporada para cada tratamiento se presenta en el Cuadro 2. La medición del diámetro de los frutos fue afectada de forma altamente significativa $(\mathrm{p} \leq 0,01)$, por los niveles de agua de riego. La prueba de rango rangos múltiples de Duncan al $5 \%$ de probabilidad, reflejo que la dosis de riego que obtuvo los mayores diámetros fue la lámina $120 \%$ ETc con una media de 5,42 cm, seguido por los tratamientos de riego $100 \%$ y $80 \%$ de la ETc con valores de 5,24 y 5,07 cm respectivamente y el menor diámetro lo observamos con el tratamiento $60 \%$ ETc con una media de $4,88 \mathrm{~cm}$. Estos resultados que resultan inferiores a los obtenidos por Esho y Saeed. (2017), quienes trabajando con tres cultivares de calabacín
(Alexandria F1, Khatoon F1 y Ardendo), y diferentes combinaciones de ácidos húmicos, reporta valores promedios de 4,16 cm. Mientras El-Mageed y Semida (2015) estudiando el efecto del régimen de riego en el hibrido Hi Tech reporta promedios 2,65 cm

\section{Peso del fruto}

El análisis de los datos peso del fruto mostró que existen diferencias altamente significativas $(\mathrm{p} \leq 0,01)$, entre los niveles de agua de riego. En la Tabla 3 se observa que la dosis de riego que arrojó los frutos más pesados fue $120 \%$ ETc con una media de $0.595 \mathrm{Kg}$, mientras que la de menor peso lo observamos con el tratamiento $60 \%$ ETc, con una media de $0.392 \mathrm{Kg}$. El cambio de lámina aplicada de riego de 120 a 60\% ETc trajo consigo una disminución del $34.12 \%$ del peso promedio del fruto. Esta investigación sobre calabacín sugiere que el estrés hídrico limita la acumulación de agua de la fruta carnosa, pero no afecta la distribución del carbono en la fruta (Mitchell et al., 1991). Con estos resultados queda demostrado que el aumento de las cantidades de riego tuvo un efecto positivo significativo en el peso del fruto de la calabaza y en los demás los componentes del rendimiento, presentándose una relación lineal positiva entre la cantidad de riego y los componentes de la calidad del fruto en este estudio. Los resultados obtenidos están en armonía con los trabajos de Yildirim y Korukcu (2000), de Abd El-Aal et al. (2008), y de Ozbahce y Tari (2010), quienes reportan que el peso de la fruta se ve afectado significativamente por los regímenes bajos de riego. También se observan resultados similares en muchos otros estudios. Amer (2011) encontró que el rendimiento de la calabaza, el peso de la fruta, el número, el diámetro y la longitud disminuyeron significativamente por la reducción 
del agua de riego aplicada según las condiciones de riego adecuadas (100\% de ETc). Ertek et al. (2004) concluyeron que las cantidades de riego tuvieron efectos significativos sobre el rendimiento y los componentes del rendimiento de la calabaza y las relaciones entre el rendimiento y los componentes del rendimiento con la cantidad de agua de riego fueron positivamente lineales. Al-Omran et al. (2005) determinaron que el rendimiento de frutos de calabaza fue mayor para condiciones de riego sin estrés ( 100 y $120 \%$ de la evapotranspiración estimada del cultivo) en comparación con las condiciones de riego estresadas (60 y 80\% de la evapotranspiración estimada del cultivo). ElGindy et al. (2009) mostraron que una mayor cantidad de riego ( $80 \%$ de ETc) proporcionó mayores rendimientos en fresco, diámetros de frutos, número de frutos por planta y longitudes de frutos en calabazas de verano regadas por goteo en comparación con aquellas con una menor cantidad de riego $(60 \%$ de ETc). Por otro lado, El-Dewiny (2011), indicó que la reducción de la cantidad de riego de 100 a $85 \%$ proporcionó un aumento en el rendimiento de la calabaza que se regó por goteo con 100, 85, 70 y 50\% de ETc. Sin embargo, el aumento de los déficits de agua redujo el rendimiento de la calabaza de verano.

\section{Efecto de la edad de trasplante sobre los compo- nentes del rendimiento del calabacín}

Como se muestra en la Tabla 4, la comparación entre tratamientos de riego se realizó a un nivel de significancia $\mathrm{p} \leq 0,05$. Los resultados de la Tabla 4 , indican que las variables número de frutos por planta, longitud y diámetro del fruto se vieron afectadas significativamente por la edad de trasplante. El análisis de varianza señala que existen diferencias significativas por efecto de las edades de trasplante. La edad de trasplante 21 días fue la que arrojó el mayor número de frutos por plantas, longitud y diámetro del fruto, con valores promedios de $22,83 \mathrm{~cm}, 20,64 \mathrm{~cm}$ y $5,19 \mathrm{~cm}$, respectivamente. En la variable peso del fruto no se observó ningún efecto de la edad de trasplante.

NeSmith (1993) también señala que la edad de 21 días es la edad ideal para trasplantar el calabacín. El crecimiento de un fruto es determinado por dos componentes: la división celular, la cual comienza desde antes de la antesis y continúa hasta los primeros días después del cuajado del fruto, y la expansión celular, responsable del aumento en tamaño, la cual es influida por la extensibilidad de la pared celular, la epidermis (y otras capas subyacentes) y por la turgencia de las células generada por el flujo de agua hacia la misma (Araujo $e t$ al., 1997). Complementariamente, este crecimiento obedece a la translocación de fotoasimilados desde las hojas y otros órganos de almacenamiento y a la presión de turgencia que se ejerce sobre las paredes celulares de las células del fruto en formación. Méndez et al. (2004) sostienen que el máximo crecimiento de los frutos, en términos de diámetro, ocurre como resultado de la elongación celulary de la acumulación de sintetizados de reserva, principalmente de sacarosa. Laguado et al. (2002) afirman que el crecimiento en grosor del fruto de guayaba (Psidium guajaba) se da rápidamente indicando un aumento significativo en la multiplicación celular dando inicio así a la etapa de elongación celular, caracterizada por un gran aumento en tamaño y siguiendo un patrón de crecimiento doble sigmoide; mientras que Mazorra et al. (2003) reportan que los cambios expresados en el diámetro del fruto de uchuva (Physalis peruviana) corresponden a una curva de tipo exponencial ajustada al modelo logístico, donde el diámetro del fruto crece constantemente sin mostrar

Tabla 4. Efecto de la edad de trasplante sobre las variables de calidad del fruto

\begin{tabular}{ccccc}
\hline $\begin{array}{c}\text { Niveles hídricos } \\
\text { de reiego (\%ETc) }\end{array}$ & $\mathbf{N}^{\circ}$ de frutos & $\begin{array}{c}\text { Longitud del fruto } \\
(\mathbf{c m})\end{array}$ & $\begin{array}{c}\text { Diámetro del } \\
\text { fruto }(\mathbf{c m})\end{array}$ & Pero del fruto (g) \\
\hline 21 & $22,83 \mathrm{a}$ & $20,64 \mathrm{a}$ & $5,19 \mathrm{a}$ & $471,25 \mathrm{a}$ \\
14 & $18,75 \mathrm{~b}$ & $20,24 \mathrm{~b}$ & $5,11 \mathrm{~b}$ & $493,34 \mathrm{a}$ \\
DMS & 1,35 & 0,318 & 0,073 & 30,2 \\
CV $(\%)$ & 17,94 & 1,65 & 1,51 & 8,05 \\
\hline
\end{tabular}

Medias seguidas por diferentes letras en una misma columna son estadísticamente diferentes a $\mathrm{p} \leq 0,05$ 
descenso hasta el final. Las diferencias en el peso de los frutos se atribuyen a la composición genética y al ambiente, pues el componente varietal tiene una gran influencia sobre la velocidad de crecimiento, el tamaño final y la forma del fruto. Esto probablemente se debe a que durante el período de crecimiento ocurre simultáneamente la acumulación de solutos y de agua en la célula, básicamente en la vacuola (basado en las propiedades de permeabilidad del tonoplasto), la cual provee espacio para el almacenamiento de solutos y la fuente de presión que actúa contra las paredes celulares causando turgencia y estiramiento del tejido (Araujo et al., 1997). Adicionalmente, Méndez et al. (2004), afirman que el tamaño final del fruto está estrechamente correlacionado con el número de semillas y de lóculos; también es afectado por la cantidad de asimilados provenientes de las hojas, la temperatura ambiental, la temperatura interna del fruto y la luminosidad. Investigaciones realizadas por Wessel (1992); Tavares et al. (1999) y Araméndiz et al. (2009) en tomate, pimentón $\mathrm{y}$ berenjena, resaltan que al aumentar al número de frutos/planta, disminuye el peso de cada fruto, por la correlación negativa entre estos dos caracteres. Por lo general, un aumento del número de frutos está asociado al plan de fertilización, que producen un mayor desarrollo vegetativo y un mayor número de inflorescencias por planta (Santos et al., 2001).

\section{CONCLUSIONES}

Los resultados de este estudio demuestran que los requerimientos hídricos y las edades de trasplante afectan de forma significativa a las variables físicas del fruto. El tratamiento de riego más efectivo resultó el de $120 \%$ ETc, indicando que el calabacín es un cultivo muy exigente en agua y su uso del agua con riego por goteo es significativamente importante para obtener una mejor calidad de frutos en el campo bajo el clima tropical de la zona de Venezuela. Se confirma el efecto negativo de las condiciones de déficit de riego sobre los atributos de calidad física. En este trabajo las variables más afectadas fueron peso y número de frutos. La edad de trasplante más recomendada es la de 21 días.
Por último, es importante hacer investigaciones en la determinación del Kc real de la berenjena para esta zona y contrastarlo con los resultados obtenidos en esta investigación.

\section{V.AGRADECIMIENTO}

Los autores quieren agradecer al Instituto Internacional de Educación (IIE) con sede en New York y al Program Officer, Scholar Rescue Fund por su apoyo para realizar esta investigación.

\section{CONTRIBUCIÓN DE LOS AUTORES}

Todos los autores participaron en la conceptualización, metodología, investigación, redacción del manuscrito inicial, revisión bibliográfica, y en la revisión y aprobación del manuscrito final.

\section{CONFLICTO DE INTERESES}

Los autores declaran no tener conflicto de intereses.

\section{REFERENCIAS BIBLIOGRÁFICAS}

Abd El-Aal, F. S; Abdel Mouty, M. M., y Ali, A. H. 2008. "Combined Effect of Irrigation Intervals and Foliar Application of Some Antitranspirants on Eggplant Growth, Fruits Yield and Its Physical and Chemical Properties". J. Agric. Biol. Sci. 4 (5): 416-423.

Abdelraouf, R. E. y M. E. Abuarab. 2012. "Effect of Irrigation Frequency under Hand Move Lateral and Solid Set Sprinkler Irrigation on Water Use Efficiency and Yield of Wheat". Journal of Applied Sciences Research 8 (11): 5445-5458.

Allen, R. G, L. S. Pereira, D. Raes, y M. Smith. 1998. Crop Evapotranspiration: Guidelines for Computing Crop Requirements: Irrig. Drain. Rome (Italy): FAO

Al-Omran, A. M., A. S. Sheta, A. M. Falatah, y A. R. Al-Harbi. 2005. "Effect of drip irrigation on squash (Cucurbita pepo) yield and water-use efficiency in sandy calcareous soils amended with clay deposits". Agric. Water Manage 73: 
43-55.

Alzoheiry, A. M. y A. M. Al-Moshileh. 2018. “Effect of Irrigation Systems and Mulching on Summer Squash (Cucurbita pepo L.) Production in Semi-Arid Areas". J. Soil Sci. and Agric. Eng. 9(12): 709-713.

Amer, K. H. 2011. "Effect of irrigation method and quantity on squash yield and quality". Agric. Water Manage. 98: 1197-1206.

Araméndiz, H., C. E. Cardona, y M. M. Espitia. 2009. "Correlaciones fenotípicas, ambientales y genéticas en berenjena". Acta Agronómica 58 (4): 285-291.

Araujo, F., S. Quintero, J. Salas, J. Villalobos, y A. Casanova. 1997. “Crecimiento y acumulación de nutrientes del fruto de Guayaba (Psidium guajava L.) del tipo "Criolla Roja” en la planicie de Maracaibo". Revista de la Facultad de Agronomía de la Universidad Central de Venezuela 19(4): 273-283.

Bafeel, S.O., A. E. Moftah 2008. "Physiological response of eggplants grown under different i r r i g t i o n regi m e t o antitransplanttreatments". Saudi J. Biol. Sci. 15 (2): 259-267.

Colak, Y. B., A. Yazar, İ. Çolak, H. Akça, y G. Duraktekin. 2015. "Evaluation of crop water stress index (CWSI) for eggplant under varying irrigation regimes using surface and subsurface drip systems". Agriculture and Agricultural Science Proeedia 4: 372-382.

El-Dewiny, C. Y. 2011. "Water and fertilizer use efficiency by squash grown understress on sandy soil treated with acrylamide hydrogels". $J$. Appl. Sci. Res. 7: 1828-1833.

El-Gindy, A. G. M., S. El-Banna El, M. A. El-Adl, y M. F. Metwally, M. F. 2009. "Effect of fertilization and irrigation water levels on summer squash yield under drip irrigation". Misr $J$. Agric. Eng. 26:94-106.

El-Habbasha, S. F., E. M. Okasha, R. E. Abdelraouf, y A. S. H. Mohammed. 2014. "Effect of pres- sured irrigation systems, deficit irrigation and fertigation rates on yield, quality and water use efficiency of groundnut". International Journal of Chem. Tech Research 1507 (01): 475-487.

El-Mageed T. A. A. y W. M. Semida. 2015. "Effect of deficit irrigation and growing seasons on plant water status, fruit yield and water use efficiency of squash under saline soil". Scientia Horticulturae 186(2015): 89-100.

Ertek, A., S. Sensoy, C. Kucukyumuk, y I. Gedik. 2004. "Irrigation frequency and amount affect yield components of summer squash (Cucurbita pepo L.)". Agric. WaterManage. 67: 63-76.

Esho K. B. y S. H. Saeed. 2017. "Effect of humic acid on growth and yield of three cultivars of summer squash (Cucurbita pepo L.). Egypt. J. Exp. Biol. (Bot.) 13 (2): 167-171.

Fernández García, I., S. Lecina, M. C. Ruiz-Sánchez, J. Vera, W. Conejero, M. R. Conesa, A. Domínguez, J.J. Pardo, B. C. Léllis, y P. Montesinos. 2020. "Trends and Challenges in Irrigation Scheduling in the Semi-Arid Area of Spain". Water 12 (3): 785

Ferreyra, R. E. y J. Jeznach. 2007. "Some maintenance problems of the drip irrigation in Poland". Annals of Warsaw University of Life Sciences - SGGW38:41-47.

Ibrahim, E. A. y E. M. Selim. 2007. "Effect of irrigation intervals and nitrogen fertilizer rates on summer squash (Cucurbita pepo L.) growth, yield, nutritional status and water use efficiency". J. Agric. Sci. Mansoura Univ. 32 (12): 10333-10345.

Ibrahim, E. A. y E. M. Selim. 2010. "Effect of irrigation intervals and antitranspirant (Kaolin) on summer squash (Cucurbita pepo L.) growth, yield, quality and economics". J. Soil Sci. and agric. Engineering, Mansoura Univ. 1 (8): 883-894.

Li, X., X. Zhang, y J. Niu. 2016. "Irrigation water 
productivity is more influenced by agronomic practice factors than by climatic factors in Hexi Corridor, Northwest China". Sci. Rep. 6: 37971 .

Gil, J. A., N. Montaño, R. Plaza. 2012. "Efecto del riego y la cobertura del suelo sobre la productividad de dos cultivares de ají dulce". Revista Biagro 24(2):143-148.

Gil, J. A., N. Montaño, y J. A. Valderrama. 2014. "Efecto de cuatro láminas de riego y dos edades de trasplante sobre el rendimiento y producción de dos cultivares de melón (Cucumis melo L.)". Revista UDO-Agricola 14 (1).

Kuslu, Y., U. Sahin, F. M. Kiziloglu, y S. Memis. 2014. "Fruit Yield and Quality, and Irrigation Water Use Efficiency of Summer Squash DripIrrigated with Different Irrigation Quantities in a Semi-Arid Agricultural Area" Journal of Integrative Agriculture 13 (11): 2518-2526.

Laguado, N., M. Marín, L. Arenas, F. Araujo, C. Castro, y A. Rincón. 2002. “Crecimiento de frutos de guayabo (Psidium guajava L.) del tipo criolla roja”. Revista Facultad de Agronomía Universidad del Zulia 19(4): 273-283.

Mazorra, M., A. Quintana, D. Miranda, G. Fisher, y B. Chávez. 2003. "Análisis sobre el desarrollo y la madurez fisiológica del fruto de uchuva en la zona de Sumapaz (Cundinamarca)”. Agronomía Colombiana 21 (3): 176-187.

Méndez, M., G. Ligarreto, M. Hernández, y L. Melgarejo. 2004. Evaluación del crecimiento y determinación de índices de cosecha en frutos de cuatro materiales de ají (Capsicum sp.) cultivados en la Amazonía colombiana. Agronomía Colombiana 22 (1): 7-17.

Montaño Mata, N. J., J. A. Gil Marín, y Y. Palmares. 2018. "Rendimiento de pepino (Cucumis sativus $L$.) en función del tipo de bandeja y la edad de trasplante de las plántulas". Anales Cientificos 79 (2): 377-385.

Mitchell, J. P., C. Shennan, y S. R. Grattan. 1991. “Developmental changes in tomato fruit composi- tion in response to water deficit and salinity". Physiologia Plantarum 83 (1): 177-185.

Moreno-Reséndez A., J. L. Reyes-Carrillo, P. Preciado-Rangel, M. G. Ramírez-Aragón, y M. R. Moncayo-Luján. 2019. "Desarrollo de calabacita (Cucurbita pepo L.) con diferentes fuentes de fertilización bajo condiciones de invernadero". Ecosist. Recur. Agropec. 6 (16): 145 151.

NeSmith, D. S. 1993. “Transplant Age Influences Summer Squash Growth and Yield". Hortscience 28 (6): 618-620.

Okasha, E. M., F. A. Hashem, y I.M. El-Metwally. 2020. "Effect of irrigation system and irrigation intervals on the water application efficiency, growth, yield, water productivity and quality of squash under clay soil conditions". Plant Archives 20 (2): 3266-3275.

Ozbahce, A. y A. F. Tari. 2010. "Effects of different emitter spaces and water stress on yield and quality of processing tomato under semi-arid climate conditions". Journal of Agricultural Water Management 97 (9): 1405-1410.

Peil, R. M. N., A. S. Strassburger, Y L. A. da Fonseca. 2012. "Growth, water consumption and use efficiency of summer squash crop in closed rice husk medium growing system". ActaHorticulturae 952: 645-650.

Pervez, M. A., C. M. Ayub, H. A. Khan, M. A. Shahid, y I. Ashraf. 2009. "Effect of drought stress on growth, yield and seed quality of tomato (Lycopersicon esculentum L.)". Pakistan Journal of Agricultural Sciences 46 (3): 174178.

Rafie, R. M. y F.M. El-Boraie. 2017. "Effect of Drip Irrigation System on Moisture and Salt Distribution Patterns under North Sinai Conditions”. Egypt. J. Soil Sci. 57 (3): 247-260.

Rolbiecki, R., S. Rolbiecki, C. Podsiadło, D. Wichrowska, A. Figas, B. Jagosz, y W. Ptach. 2017. "Influence of drip irrigation on the yielding of summer squash "White Bush" 
under rainfall-thermal conditions of Bydgoszcz and Stargard". Infrastruktura, Ekologia Terenów Wiejskich 3 (2): 12291240.

Santos, G. M., A. P. Oliveira, J. A. L. Silva, E. U. Alves, y C. C. Costa. 2001. “Características e rendimento de vagem do feijão-vagem em função de fontes e doses de materia organica". HorticulturaBrasileira 19(1): 30-34.

Tavares, M., A. De Melo, y W. Bueno. 1999. "Efeito diretos e indiretos e correlações canônicas para caracteres relacionados com a produção de pimentão". Bragantia 58 (1): 41-47.

Tian, F., P. Yang, H. Hu, y H. Liu. 2017. "Energy balance and canopy conductance for a cotton field under film mulched drip irrigation in an arid region of northwestern China". Agric. Water Manage. 179: 110-121.

Wetzel J. y Stone A. 2019. "Yield Response of Winter Squash to Irrigation Regime and Planting Density”. Hortscience 54 (7): 1190-1198.

Wessel-Beaver, L. 1992. “Genetic variability of fruit set, fruit weight, and yield in a tomato population grown in two high-temperature environments". Journal of the American Society for Horticultural Science 117 (5): 867-870.

Xu, C. y D. I. Leskovar. 2014. "Growth, physiology and yield responses of cabbage to deficit irrigation". Hort. Sci. 41: 138-146.

Yavuz, D., M. Seymen, N. Yavuz, y Ö. Türkmen. 2015. "Effects of irrigation interval and quantity on the yield and quality of confectionary pumpkin grown under field conditions". Agricultural Water Management 159: 290-298.

Yildirim O., y A. Korukcu. 2000. Comparison of Drip, Sprinkler and Surface Irrigation Systems in Orchards. Ankara (Turkey): University of Ankara.

Zwart, S. J. y W.G.M. Bastiaanssen. 2004. "Review of measured crop water productivity values for irrigated wheat, rice, cotton and maize". Agricultural Water Management 69: 115-133. 